\title{
ELECTROMAGNETIC WAVE SCATTERING EXPERIMENTS IN HALL THRUSTER PLASMA PLUMES
}

\author{
Brian E. Gilchrist $\stackrel{+}{\ddagger}$, Christopher N. Davis*, Douglas O. Carlson*, and Alec D. Gallimore \\ University of Michigan \\ Ann Arbor, MI
}

\begin{abstract}
$\underline{\text { Abstract }}$
We report on electromagnetic wave (EM) scattering experiments using a $17 \mathrm{GHz}$ signal propagated through an electric propulsion Hall thruster plume at different radial and axial distances. The goals of the experiments were to evaluate the spectral modification of the EM signals after propagating through the plume. These spectral modifications are attributed to temporal variations in the plasma due to propagating density perturbations. The experiments were performed on a D-55 Hall thruster manufactured by TNIIMASH (Research Institute of Machine Building) and the University of Michigan $5 \mathrm{~kW}$ Hall thruster. Close to the exit plane on the D- 55 thruster axis, the spectral response is dominated by a first sideband at approximately $+/-100 \mathrm{kHz}$ with respect to the $17 \mathrm{GHz}$ signal. Further downstream in the axial direction, first and second sidebands are both present for the D-55. The spectral response is not distinguishable above the baseline noise beyond $+1-300 \mathrm{kHz}$ with respect to the signal. For the UM thruster, only first sidebands at $+/-12 \mathrm{kHz}$ were observed as a function of axial distance. We show that present models do not adequately predict the observed axial variations or sideband levels. A revised model that assumes a rotating Gaussian density perturbation and which is assumed generated by azimuthal drift wave oscillations is shown to allow better matching of sideband levels and their variations along the plume axis.
\end{abstract}

\begin{tabular}{|c|c|c|}
\hline \multicolumn{3}{|c|}{ Nomenclature } \\
\hline & & Modulation frequency (rad) \\
\hline$E$ & Electric field $(\mathrm{V} / \mathrm{m})$ & Nth order Bessel function \\
\hline & Wave number $\left(\mathrm{m}^{-1}\right)$ & carrier frequency (rad) \\
\hline$m_{\mathrm{amp}}, r$ & $n_{\text {freq }}, \quad$ Modulation Factors for amplitude & Instability height (unitless) \\
\hline$m_{\text {phase }}$ & $\begin{array}{l}\text { (unitless), frequency (rad/s), phase (rad), and } \\
\text { density noise or oscillations (unitless) }\end{array}$ & $\begin{array}{l}\text { Distance from center of plasma to instability } \\
(\mathrm{cm})\end{array}$ \\
\hline$n_{e}$ & Electron number density $\left(\mathrm{cm}^{-3}\right)$ & \\
\hline$n_{c}$ & Critical electron density $\left(\mathrm{cm}^{-3}\right)$ & \\
\hline$n_{e}(x, y, t)$ & $\begin{array}{l}\text { Electron density as a function of time and } \\
\text { space at a fixed axial position }\left(\mathrm{cm}^{-3}\right)\end{array}$ & $1 \cdot$ Introduction \\
\hline$n_{e(x, y)}$ & $\begin{array}{l}\text { Electron density as a function of space at a } \\
\text { fixed axial position }\left(\mathrm{cm}^{-3}\right)\end{array}$ & $\begin{array}{l}\text { Recently, there have been results reported on the } \\
\text { use of microwave interferometers as highly accurate, }\end{array}$ \\
\hline & Radial distance from & non-intrusive diagnostic tools in the plumes of Hall \\
\hline & Axial distance from thruster & and arcjet electric propulsion thrusters $[1,2]$. Other \\
\hline & Position of ray & studies have measured and modeled the impact of \\
\hline$t$ & Time (s) & microwave signal propagation through these plasma \\
\hline$\phi_{\text {vacuum }}$ & Phase shift due to free space & $\begin{array}{l}\text { plumes to assess their impact on spacecraft } \\
\text { communication, radar, or radio navigation systems }\end{array}$ \\
\hline$\phi_{\text {plasma }}$ & Phase shift due to plasma & {$[2,3]$. In general, it has been shown that for present } \\
\hline & $\begin{array}{l}\text { Phase shift of propagated wave relative to } \\
\text { phase with out a plasma }\end{array}$ & $\begin{array}{l}\text { day, kilowatt-class Hall thrusters, significant } \\
\text { attenuation is primarily observed in the lower }\end{array}$ \\
\hline$\phi(t)$ & Phase of wave at time, $t$ (rad) & microwave frequency bands (below about $8 \mathrm{GHz}$ ) \\
\hline$\lambda$ & Wavelength $(\mathrm{m})$ & spreading effects $[1,2]$ \\
\hline & $\begin{array}{l}\text { Standard deviation (width) of azimuthal } \\
\text { - instability }(\mathrm{cm})\end{array}$ & $\begin{array}{l}\text { However, phase modification of electromagnetic wave } \\
\text { (EM) signals is significant throughout the microwave }\end{array}$ \\
\hline$\omega$ & Radial frequency $(\mathrm{rad} / \mathrm{sec})$ & $\begin{array}{l}\text { frequency range to above } 20 \mathrm{GHz} \text { and well out in the } \\
\text { plume [2]. Further, both amplitude and phase }\end{array}$ \\
\hline $\begin{array}{l}\text { Associate } \\
\text { senior mer }\end{array}$ & $\begin{array}{l}\text { Professor, Electrical Engineering and Computer Science, } \\
\text { mber AIAA }\end{array}$ & $\begin{array}{l}\text { temporal variations can generate frequency sidebands } \\
\text { on microwave EM signals propagating through }\end{array}$ \\
\hline $\begin{array}{l}\text { Gradua } \\
\text { Comput }\end{array}$ & $\begin{array}{l}\text { Student Research Assistant, Electrical Engineering and } \\
\text { I Science, member AIAA }\end{array}$ & $\begin{array}{l}\text { plumes such as seen in Figure } 1 \text { for an SPT- } 100 \text { Hall } \\
\text { thruster }[2,3] \text {. These effectswill become even more }\end{array}$ \\
\hline
\end{tabular}


significant as power levels for Hall thrusters increase.

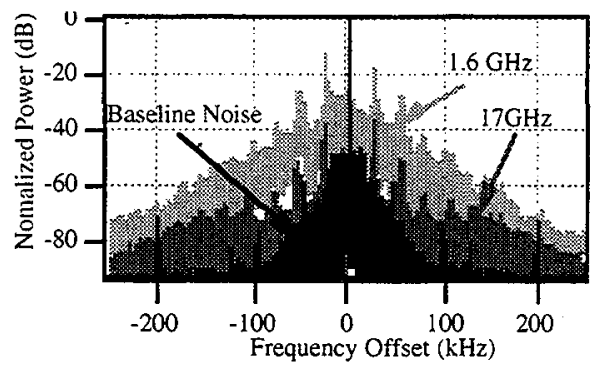

Figure 1 - Spectral response of the SPT-100 at 1.6 and $17 \mathrm{GHz}$ [3].

In much of this work to-date, an assumption of azimuthal symmetry in the plasma plume has been made. This appears to be a good approximation for thrusters studied where time-averaging of measurements is used $[4,2]$. However, here we wish to consider the effect of important plasma oscillations in Hall thrusters and their introduction of asymmetric azimuthal temporal variations in the Hall thruster plasma plume. Specifically, we focus on coherent azimuthal drift waves which, along with other more stochastic disturbances, were reviewed by Choueiri [5]. They were also the focus of recent in-situ experimental measurements of Hall thruster transient behavior reported by Hargus et al. [6].

Below, in Section 2 we review present temporal models describing EM signal propagation through plasma plumes which assumes azimuthal symmetry and also briefly summarize known plasma instabilities in Hall thrusters. In Section 3 we describe our experimental set-up for measurements (described in Section 5) which are used to demonstrate the limitations of existing models as described in Section 4. Section 5 will present a revised model that assumes the oscillations are rotating azimuthal plasma disturbances and that they show good agreement with observations. We will conclude with a discussion and summary in Section 6 of these results.

\section{Theoretical Background}

\section{Electromagnetic Wave Interactions}

A plane wave propagating through a plasma can be modulated both in phase and in amplitude. The following is a mathematical summary of amplitude and phase modulation

Suppose we have a plane wave

$$
E(r)=E_{o}(\bar{r}) \cos \left(w_{o} t\right)
$$

Pure amplitude modulation is represented as

$$
E(\bar{r})=E_{o}(\bar{r}) \cos \left(w_{o} t\right)\left(1+m_{a m p} \cos \left(w_{m} t\right)\right)
$$

which can be expanded via a well known trigonometric identity to

$$
\begin{aligned}
E(\bar{r})= & E_{o}\left(\cos \left(w_{o} t\right)+\frac{m_{a m p}}{2}\left[\cos \left(\left(w_{o}+w_{m}\right) t\right)\right.\right. \\
& \left.+\cos \left(\left(w_{o}-w_{m}\right) t\right)\right]
\end{aligned}
$$

Here $m_{a m p}$ is a modulation fraction and $\omega_{m}$ is the modulation frequency. In the above expression we see explicitly contributions from the original signal plus the first sidebands.

Similarly, using phasor notation, pure phase modulation may be represented by

$$
E(\bar{r})=E_{o}(\bar{r}) \exp \left(j\left(w_{o} t+m_{\text {phase }} \cos \left(w_{p} t\right)\right)\right)
$$

Here $m_{\text {phase }}$ is a modulation fraction and $\omega_{p}$ is the modulation frequency. Recall when using phasor notation, to obtain physical field we must take the real part of $E(r)$. The frequency spectrum may be obtained by expanding the exponential in terms of Bessel functions of the first kind. We use the following identity,

$$
\exp (j x \cos (\theta))=\sum_{n=-\infty}^{\infty} j^{n} J_{n}(x) \cos (n \theta)
$$

where $J_{n}(x)$ is the n-th Bessel function. Expanding and taking the real part yields

$$
\begin{aligned}
& E(\bar{r})=E_{o}(\bar{r})\left(J_{o}\left(m_{\text {phase }}\right) \cos \left(w_{o} t\right)\right. \\
& -J_{1}\left(m_{\text {phase }}\right)\left(\sin \left(w_{o}+w_{p}\right) t+\sin \left(w_{o}-w_{p}\right) t\right) \\
& +J_{2}\left(m_{\text {phase }}\right)\left(\cos \left(w_{o}+2 w_{p}\right) t-\cos \left(w_{o}-2 w_{p}\right) t\right) \\
& +\ldots)
\end{aligned}
$$

Whereas pure amplitude modulation gives rise only to the first harmonics, pure phase modulation gives rise to an infinitude of harmonics. If the argument m_phase is less than one, the magnitude of the Bessel function decrease as the frequency of the harmonics increase.

\section{Hall Thruster Instability Summary}

Here we briefly summarize the kinds of oscillations that occur inside the SPT acceleration channel. A more detailed review has been given by Choueiri [5]. As the ions and their associated electrons are accelerated into the plume, time variation in the local electron density in the plume may be affected. The oscillations include low frequency azimuthal drift waves, axially propagating "transit-time" oscillations, high frequency azimuth drift waves, ionization instability waves and wave emission associated with weakly ionized, inhomogeneous plasma in crossed electric and magnetic fields.

In addition to Hall thruster type, operating conditions of the thruster also affect the frequency range characteristic of different instabilities. For the instabilities below, the general operating conditions are as follows [5]: diameter of acceleration channel $(\sim 10 \mathrm{~cm})$, discharge voltage $\left(U_{d}=200 \mathrm{~V}\right)$, mass flow 
rate $(\mathrm{dm} / \mathrm{dt}=3 \mathrm{~g} / \mathrm{s}$ of $\mathrm{Xe})$, and, discharge current $\left(\mathrm{I}_{\mathrm{d}}\right.$ $=3-3.2 \mathrm{~A}$ ).

Oscillations between 1 and $20 \mathrm{kHz}$ are sensitive to the circuitry of the thruster and are relatively non existent under nominal operating conditions. Between 20 and $60 \mathrm{kHz}$ a type of low frequency azimuthal propagating wave is present $[5,6]$. These are excited predominantly near the exit plane of the thruster in the vicinity of negative gradient of the mostly radial magnetic field $(\mathrm{dBr} / \mathrm{d} z<0$, where $\mathrm{dz}$ is along the axial direction). The strength of these waves is dependent on the operation region (I-V curve). For instance, these waves are dominant at low discharge voltage $U_{d}$ and diminish in the current saturation region.

Between 20 and $100 \mathrm{kHz}$ exist axially propagating waves which are bounded by the ion collision frequency $(-20 \mathrm{kHz})$ and the electron collision frequency $(-200 \mathrm{kHz})$. Contained in this region is the ionization frequency $(\sim 35 \mathrm{kHz})$. Oscillations between 70 and $500 \mathrm{kHz}$ also known as "transit-time" oscillations are turbulent [7]. The frequency is related to ion velocity $v_{0}$ and the length of the acceleration channel $L$, by $f=v_{0} / L$. Finally there exist a host of high frequency oscillations where $\mathrm{f}>0.5 \mathrm{MHz}$. These tend to dominate during nominal operation of the thruster. One type of high frequency oscillation is mostly azimuthal and was the first to be predicted theoretically [8]. Besides this type other high frequency oscillations have not been well studied.

\section{3•Experimental Set-Up}

A $17 \mathrm{GHz}$ microwave measurement system [1] has been used to measure the spectral power distribution through the Xenon plasma exhaust of two Hall Thrusters. These were the model D-55 anode layer thruster developed by TsNIIMASH, which was on loan from the Jet Propulsion Laboratory, and the UM $5 \mathrm{~kW}$ Hall thruster designed at the University of Michigan. The D-55 was operated at $300 \mathrm{~V}$ and 4.5 A $(1.35 \mathrm{~kW})$. The UM thruster was operated at 500 $\mathrm{V}$ and $10.3 \mathrm{~A}(5 \mathrm{~kW})$.

Figure 2 shows a schematic of the experimental setup. This system consisted of a computer controlled network analyzer, a spectrum analyzer connected to a microwave up-down frequency conversion circuit, two lens corrected horn antennas with a beamwidth of about 7 to 8 degrees, and a computerized positioning table. The network analyzer was used as the-signal source. The experimental measurements were all performed at the Plasmadynamics and Electric Propulsion Laboratory (PEPL) of the University of Michigan in its $6 \mathrm{~m}$ by $9 \mathrm{~m}$ stainless steel vacuum chamber. The pressure during testing was about $5 * 10^{-5}$ Torr.

All components except the spectrum analyzer, and computers were placed in the vacuum chamber.
The assembled microwave system mounted onto a positioning table which was able to move up to $.9 \mathrm{~m}$ axially from the thruster plane and up to $1.5 \mathrm{~m}$ radially allowing for measurements at several axial and radial positions within the thruster plume.

The two thrusters used in this experiment were a model D-55 anode layer thruster developed by TsNIIMASH, which was on loan from the Jet Propulsion Laboratory, and the UM thruster a $5 \mathrm{~kW}$ Hall thruster designed at the University of Michigan. The D-55 was operated at $300 \mathrm{~V}$ and $4.5 \mathrm{~A}$ (1.35 $\mathrm{kW}$ ). The UM thruster was operated at $500 \mathrm{~V}$ and $10.3 \mathrm{~A}(5 \mathrm{~kW})$. Both used Xenon as its primary propellant.

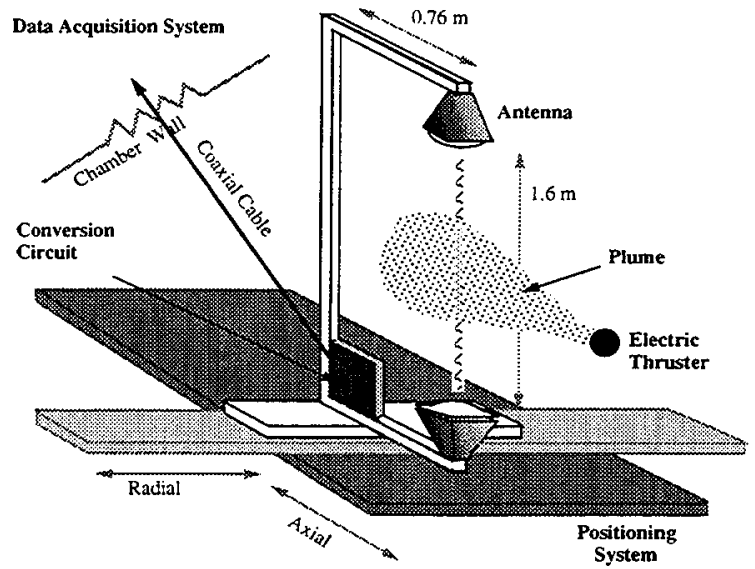

Figure 2 - Diagram of experimental setup inside of vacuum chamber used to measure spectrum of D-55 and UM thrusters.

\section{$\underline{4 \cdot \text { Experimental Results }}$}

The following figures show the measured spectral power distributions for a signal propagated through the plume of a D-55 thruster and the UM thruster at several axial positions. The transmitted signal is denoted by the solid curve and the background (no plasma present) as dashed curves. The notation $(r, z)=(0,4.75)$ [in] means that $r=0$ and $z=4.75$ inches are the radial (off-axis) and axial positions, respectively, of the measurements.

As seen in Figure 3, at 4.75 inches from the exit plane of the D-55 thruster, the signal is modulated by a broad spectrum peaked at $100 \mathrm{kHz}$. At 10 inches and beyond second order harmonics at $200 \mathrm{kHz}$ are clearly visible. At 40 inches the signal response to the plasma plume approaches the background.

As seen in Figure 4, at 10 inches from the UM thruster there are clear harmonics at $12 \mathrm{kHz}$ that are narrower and stronger than in the D-55. No new sidebands are present further out from the exit plane, and at 31 inches from the exit plane the signal 
response due to the plasma plume approaches the background.

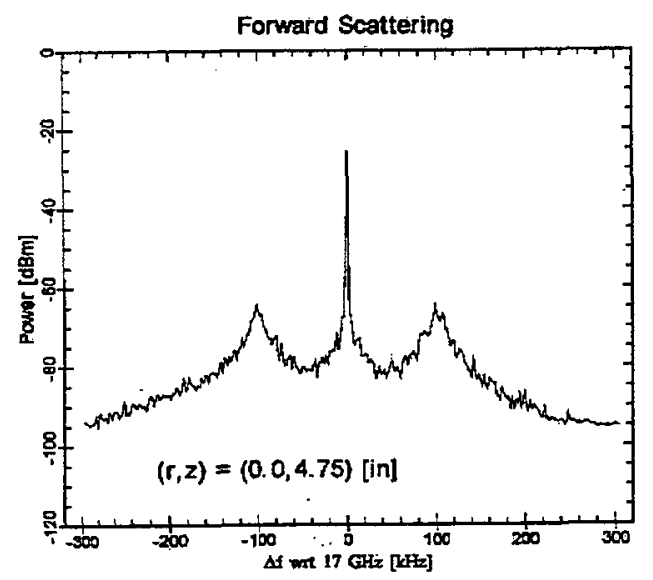

$3 a$

Forward Scattering

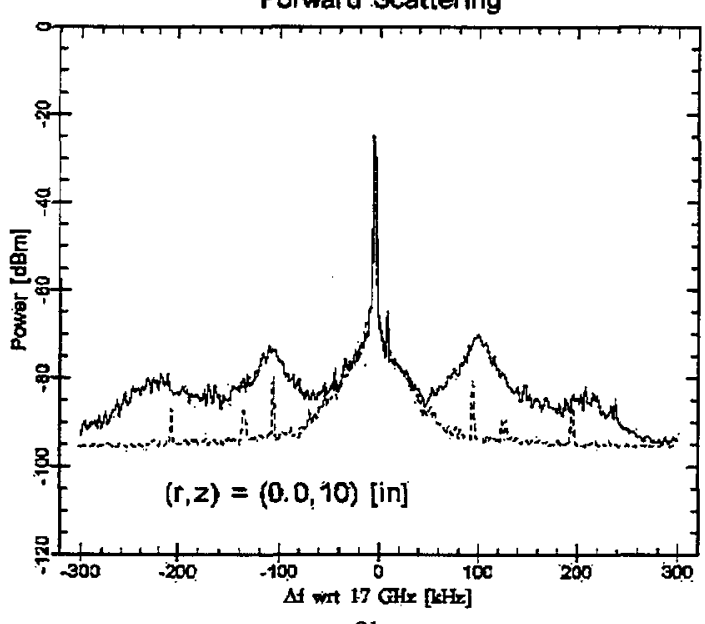

$3 b$

Forward Scattering

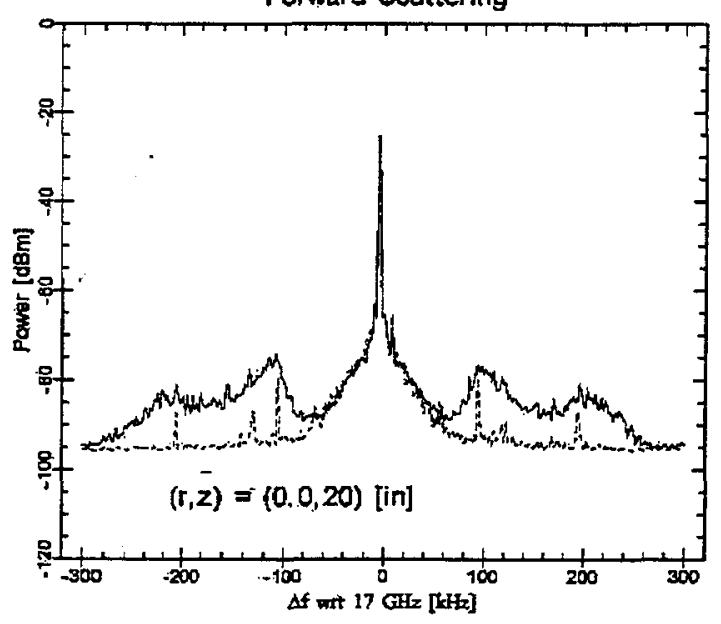

$3 c$

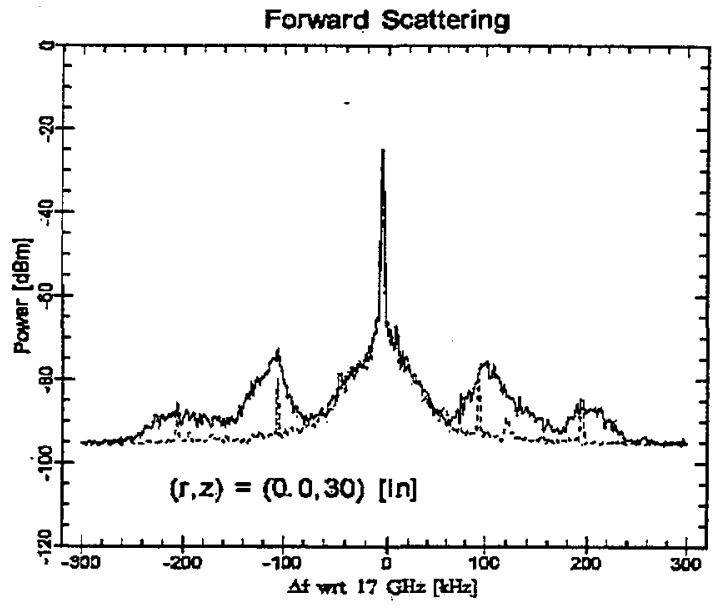

$3 d$

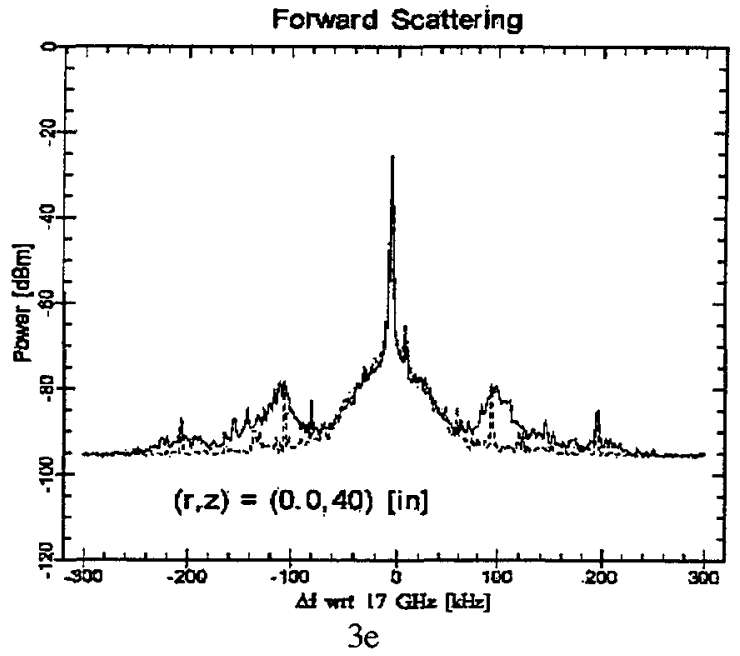

Figure 3 - Measured spectrum of a D-55 thruster at $4.75,10,20,30$, and 40 inches from the exit plane.

It should be noted that the background contains spurious signal most prominently at $100 \mathrm{kHz}$ intervals. These are due to the local oscillator in the up-down frequency conversion circuit. We argue the fact that the spurious signals correspond to the harmonics in the D-55 plasma plume is coincidental and these do not significantly contribute to the signal for the following reasons. First, the modulation of the signal is caused by a much broader array of instabilities in the plasma centered at $100 \mathrm{kHz}$ intervals. Second, close to the exit plane of the thruster the spurious signal at $200 \mathrm{kHz}$ does not contribute to the spectrum of the signal close to the exit plane of the D-55. Lastly, in the UM thruster there were no observed effects due to the plasma at harmonics of $100 \mathrm{kHz}$. 


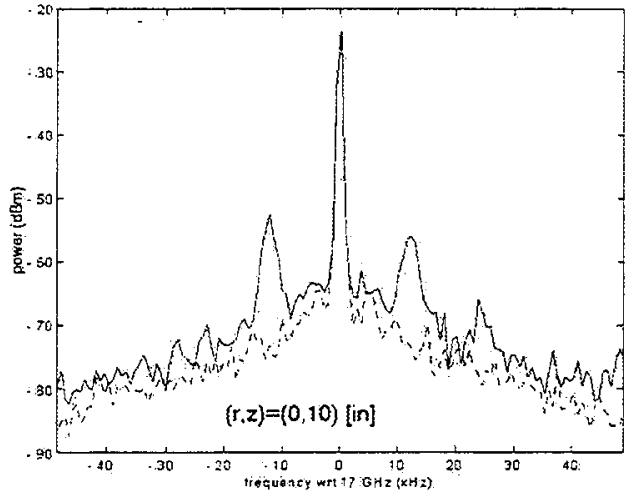

$4 \mathrm{a}$
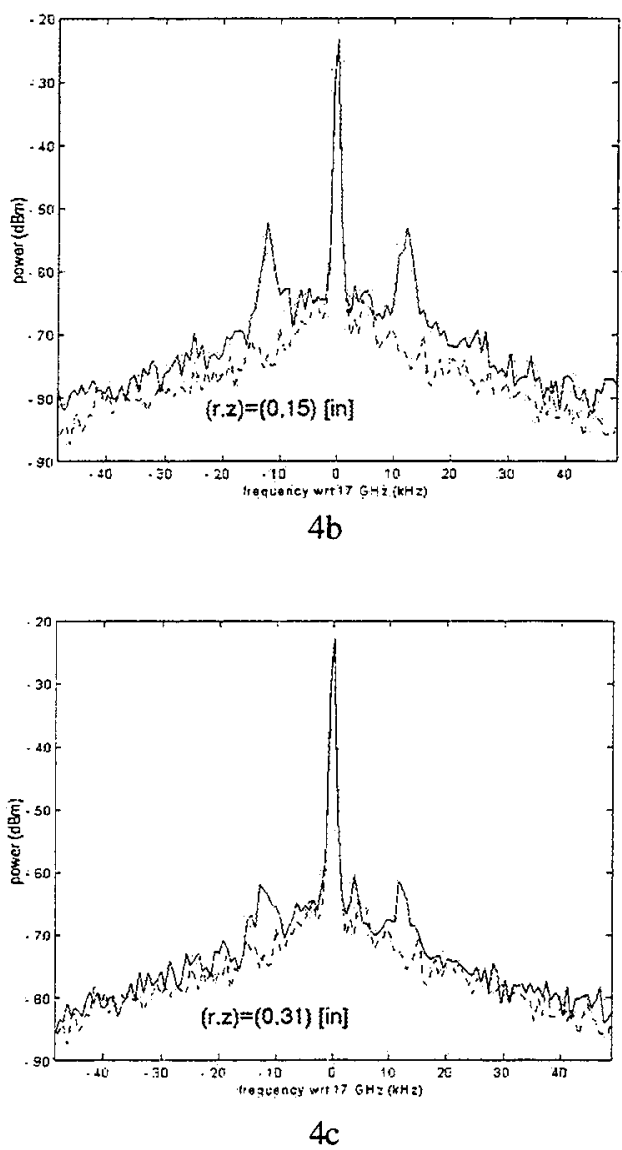

Figure 4 - Spectrum measurement for the UM Thruster at 10,15, and 31 inches from the exit plane

\section{5*Alternate Model and Results}

\section{Alternate Model}

Electromagnetic signals are known to interact with a plasma by altering the phase, amplitude, direction, and power spectral density of a transmitted signal as described in the equation below.

$$
\begin{aligned}
& E=E_{o}\left[1+m_{a m p}(t)\right] \times \\
& \cos \left[k r-\left(w-m_{\text {freq }}(t)\right) t-m_{\text {phase }}(t)\right]
\end{aligned}
$$

Equation 7 is the most general form of modulation where $\mathrm{m}_{\mathrm{amp}}, \mathrm{m}_{\text {freq }}$, and $\mathrm{m}_{\text {phase }}$ correspond to the modulation coefficients for amplitude, frequency, and phase.

In order to model the spectral responses of the plasmas that were observed, an electromagnetic signal with only phase modulation was assumed.

$$
E=E_{O} \cos (w t-\Delta \phi(t))
$$

where $k$ is equal to the free space wavelength, $\omega$ is the frequency of the signal, and $\phi$ is the phase variation of the signal due to the plasma.

The total phase shift as function of time for a signal passing through a plasma can be found from the electron density of the plasma with the following relation

$$
\begin{aligned}
\Delta \phi(t) & =\phi_{\text {vacuum }}-\phi_{\text {plasma }}(t) \\
& =\frac{w}{2 c n_{c}} \int_{s !}^{s 2} n_{e}(x, y, t) d s
\end{aligned}
$$

We estimated the electron density as a function space and time based on an azimuthal instability in the thruster's plume that was superimposed on the thruster's static (time-averaged) electron density profile. We also assumed that the azimuthal instability rotated around the center of the plume at a certain constant angular velocity, $\omega$. Figure 5 shows a three dimensional view of the plasma and the instability. We modeled the instability as a Gaussian distribution with a width, $\sigma$, height, $m$, and distance from the center of the plume, $d$, as shown in Figure 6. The total electron density (without rotation taken into account) can then be shown mathematically as

$$
n(x, y)=n(x, y) \times\left(1+m e^{-\sigma\left((x-d)^{2}+(y-d)^{2}\right)}\right)
$$

where $n(x, y)$ is the static electron distribution at a given axial position and is symmetric about the origin of the plasma plume. For the purposes of our model, we assume the static electron density also has a Gaussian distribution with a $\sigma$ equal to $7.5 \mathrm{~cm}$ and a peak density of $10^{11} \mathrm{~cm}^{-3}$.

With the assumption that the time scale of the rotation of the instability is much longer than the time scale of the signal propagating across the plasma, a computer code was generated to solve Equation 9. The instability was numerically rotated around the center of the static plume distribution at a frequency $\omega$, and at each time increment a plane wave was sent through the plasma and Equation 9 was used to calculate the phase shift of the propagated wave giving $\phi$ as a function of time. Equation 8 could then be used to give the spectrum of the signal by simply 
taking its Fourier transform. A summary of these results are given below.

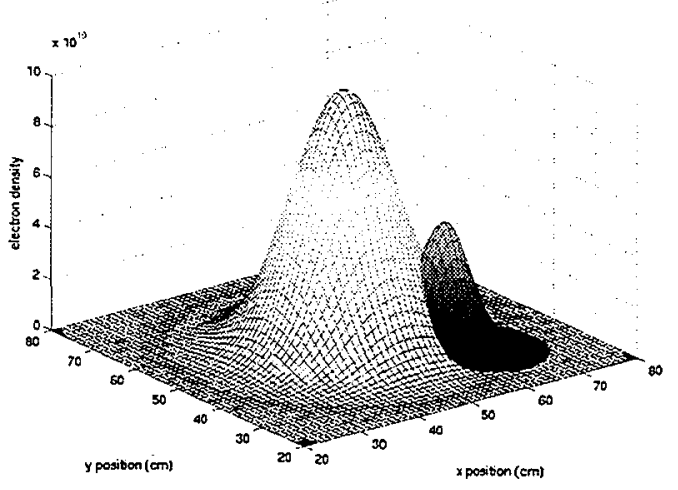

Figure 5 - 3-dimensional view of static electron density and the azimuthal instability

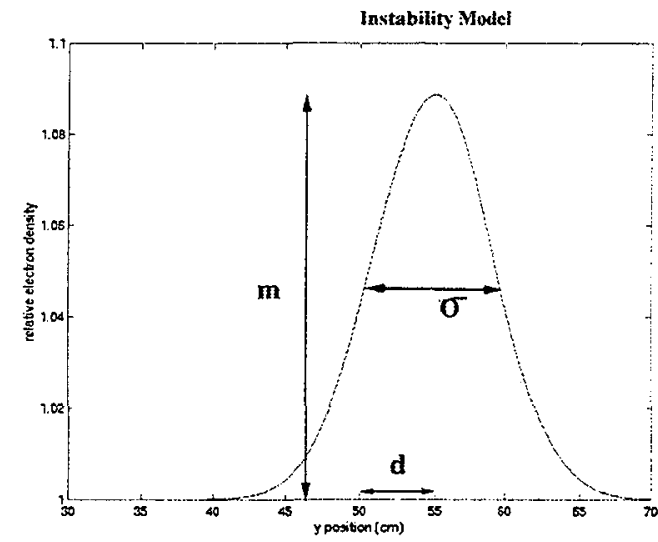

Figure 6 - 2-dimensional representation of the azimuthal instability model where $m$ is the amplitude of the instability, $\sigma$ is the width, and $\mathrm{d}$ is the distance the instability is from the origin.

\section{Comparison With Measurements}

The two sets of figures below show the comparison of the spectrum generated by Equations 5, 6 , and 7 to that of the measured spectrum. In the D55 thruster two different spectrums were observed depending of the axial positions while in the UM thruster only one spectrum was observed. All calculations were done slightly off-center of the plasma plume due to the fact that calculations near the center caused double harmonics to appear.

It was found that an oscillation with a rotational frequency of $100 \mathrm{kHz}$, height $(\mathrm{m})$ of .06 , distance from the center (d) of $3 \mathrm{~cm}$, and a $\sigma$ of $4.1 \mathrm{~cm}$ gave sidebands very close in amplitude to those observed in the measured spectrum at 10 inches from the exit plane of the D-55 thruster. The simulation results are compared with measurements in Figure $7 a$ and $b$.

We also generated a simulated spectrum with sideband amplitudes close to those measured in the near field, 4.75 inches, of the D-55 by changing the parameters of the instability as shown in Figure $8 \mathrm{a}$ and $b$. By setting the rotational frequency of the azimuthal oscillation to $100 \mathrm{kHz}$, its height (m) to .1 , the distance from the origin to $3 \mathrm{~cm}$, and $\sigma$ to 10 $\mathrm{cm}$ it was possible to simulate sidebands very close in amplitude to those observed in the measured spectrum.

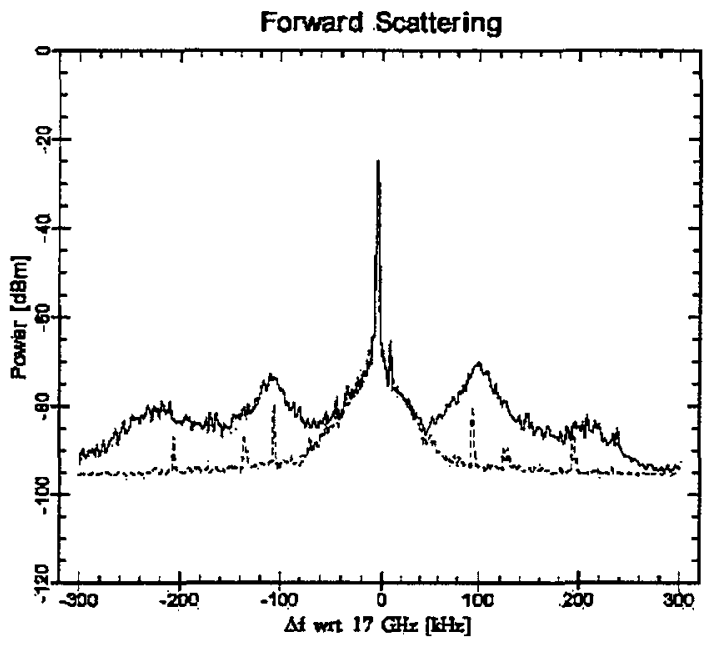

Figure 7a-Measured spectrum of the far field D55 plasma plume.

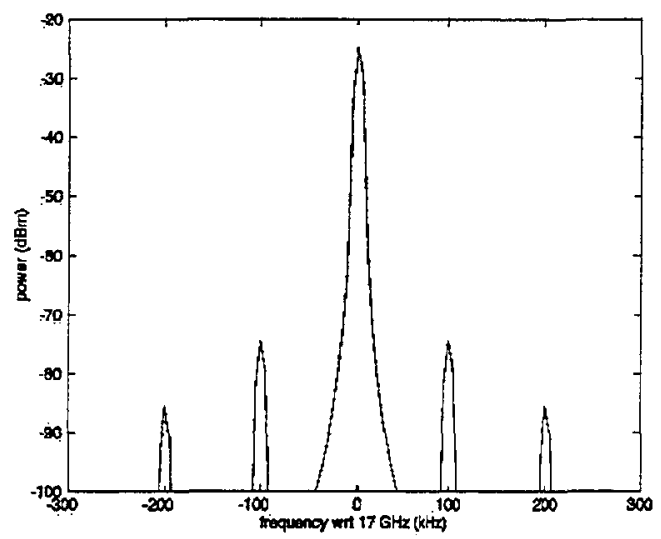

Figure $7 b$ - Theoretical spectrum for the far field D-55 plasma plume

Based on these results, there is an indication that an azimuthal instability in a D-55 thruster tends to become more narrow and lower in amplitude downstream of the thruster's exit plane.

It was also possible to reproduce the sideband levels for the UM thruster as shown in Figure $9 \mathrm{a}$ and b. Setting the height of the instability to 0.3 , the width to $10 \mathrm{~cm}$, the distance from center to $3 \mathrm{~cm}$, and the instabilities frequency of rotation to $12 \mathrm{kHz}$, we were able to obtain a spectrum with sidebands very close in amplitude to the measured spectrum. Unlike the D-55, the UM spectrum stays relatively constant down stream of the exit plane. 


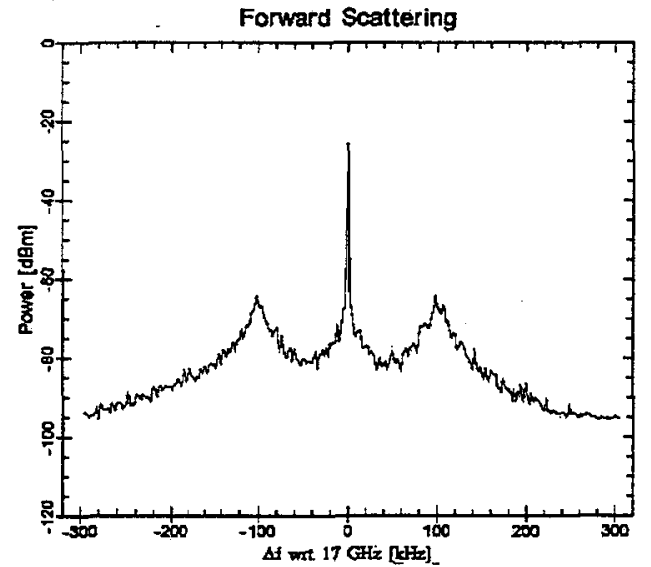

Figure 8a-Measured spectrum for the near field D-55 plasma plume.

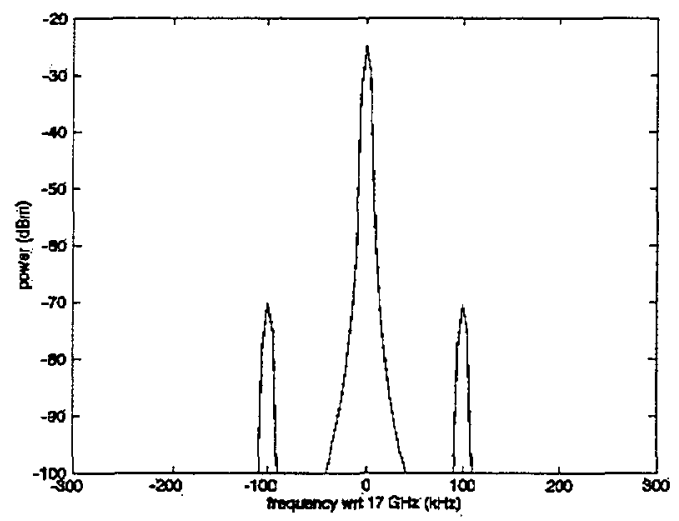

Figure $8 b$ - Theoretical spectrum for the near field D-55 plasma plume.

\section{6*Discussion and Summary}

Based on the theoretical modeling of an azimuthal instability by the methods described the following trends can be inferred. As the width of the instability increases the relative amplitudes of the sidebands increases. As the height of the instability increases, the amplitude of all the sidebands increases, but the relative amplitudes of the sidebands remain constant. And, finally, as the distance the azimuthal instability is away from the center of the plume is varied different sidebands become more or less pronounced. Therefore, we believe, it is possible to predict the shape and amplitude of a azimuthal instability by- analyzing the relative and absolute amplitudes of the sideband present in the forward scattered spectrum of a plasma thruster.

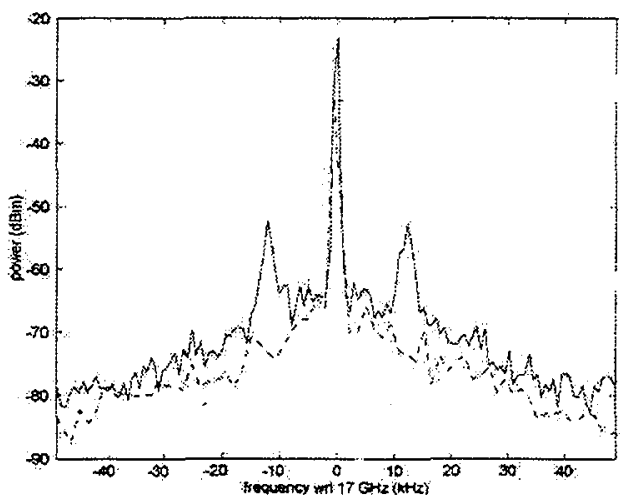

Figure 9a - Measured spectrum for the UM Thruster plasma plume.

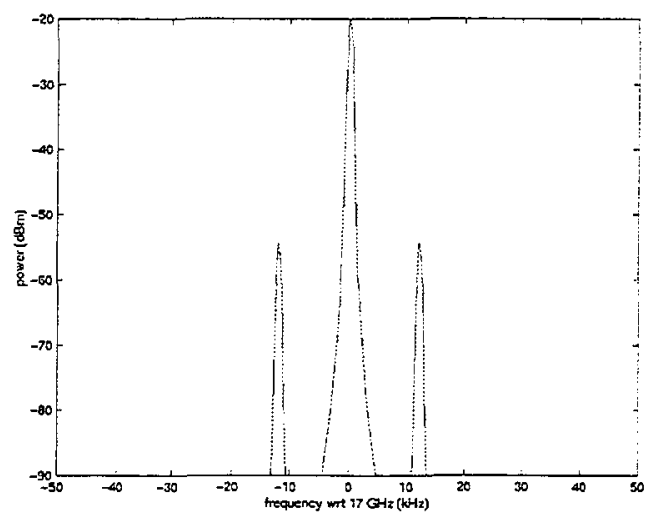

Figure $9 \mathrm{~b}$ - Calculated spectrum for the UM Thruster plasma plume.

Some known limitations of this model are the following. First, amplitude modulation due to beam spreading is not taken into account. Second, the antenna distribution needs to be taken into account instead of assuming a single plane wave. Thirdly, due to the symmetry of the electron density model used, spectral calculations done close to the middle of the plasma plume produce harmonics at double the instability rotational frequency.

In the above, we have noted that experimental measurements of frequency sidebands do not fully agree with simulations using existing models. We have proposed an alternative model that is based on known oscillation instabilities. The simulated results are more closely able to account for the observed variations.

\section{Acknowledgments}

This research was funded in part by AFOSR grant \#F49620-95-1-0331 (contract monitor: Dr. M. Birkan). The authors would like to thank the students of the PEPL lab for their efforts to operate the thrusters and chamber systems. 


\section{Bibliography}

1. Gilchrist,-B. E., S. G. Ohler, A. D. Gallimore "Flexible Microwave System to Measure the Electron Number Density and Quantify the Communications Impact of Electric Thruster Plasma Plumes," Rev. Sci. Inst., Vol. 68, No. 2, February, 1997.

2. Ohler, S.G., Ph.D. Thesis, Space Electric Propulsion Plasma Characterization Using Microwave and Ion Acoustic Wave Propagation, University of Michigan, 1996.

3. Ohler, S.G., et al., RF Signal Impact Study of an SPT, paper AIAA 96-2706 presented at 32nd Joint Propulsion Conference, Lake Buena Vista, FL, 1996.

4. Ohler, S.G., Gilchrist, B.E., and Gallimore, A.D., Non-intrusive electron number density measurements in the plume of a $I \mathrm{KW}$ arcjet using a modern microwave interferometer. IEEE Transactions on Plasma Science, 23(3), p. 428435, June, 1995.

5. Choueiri, E. Y., "Characterization of Oscillations in Closed Drift Thrusters," AIAA94-3013, 30 $0^{\text {th }}$ Joint Propulsion Conference, Indianapolis, IN, June 27-29, 1994.

6. Hargus, W. A., N. Meezan, and M. A. Cappelli, "A study of a low power Hall thruster transient behavior," IEPC $97-058,25^{\text {th }}$ International Electric Propulsion Conference, Cleveland, $\mathrm{OH}$, August 24-28, 1997.

7. Esipchuck, Y. B., A. Morozov, G. Tilinin, and A. Trofimov, "Plasma Oscillations in Closed Drift accelerators with an extended acceleration zone," Soviet Physics, Technical Physics, 18(7):928-932, 1974.

8. Esipchuck and G. Tilinin, "Plasma Oscillations in Closed Drift accelerators with an extended acceleration zone," Soviet Physics, Technical Physics, 21(4):417-423, 1976. 